\title{
Evaluation of automated large-scale screening tests for syphilis
}

\author{
D. E. MACFARLANE, KAY HARE, AND T. F. ELIAS-JONES \\ From The City Laboratory, Greater Glasgow Health Board, Glasgow G1 1RN
}

SYNOPSIS Two methods of performing serological screening tests for syphilis are compared. One consisted of the Venereal Diseases Reference Laboratory (VDRL) slide test, the cardiolipin Wassermann reaction (CWR), and the Reiter protein complement fixation test (RPCFT) performed manually; the other was a fully automated system using two Technicon AutoAnalyzers (AAII), one for the automated reagin test (ART) and the other for automated complement fixation tests. The absorbed fluorescent treponemal antibody test (FTA-ABS) was used as a final arbiter in all cases found to be seropositive by either method. A pooled antigen consisting of a mixture of cardiolipin and Reiter protein was used for the automated complement fixation test, thus increasing the scope and capacity of the system. The AutoAnalyzer was shown to be capable of performing 400 cardiolipin and Reiter complement fixation tests and 700 automated reagin tests in an 8-hour day. Modification of the complement fixation test method to take advantage of the highly sensitive colorimeter resulted in a significant increase in sensitivity and a corresponding saving in reagents. Of the 7843 sera tested, 258 gave a positive result in one or more of the screening tests.

The automated test detected many more Reiter positive sera (127) than the manual test (83). Conversely, fewer CWR positive sera were detected by the automated test $(60)$ than by the manual test (82). There was little difference between the number of positive sera detected by the ART (73) and the VDRL slide test (71). In 19 instances the automated tests detected positive sera which registered as completely negative in the manual tests, and four seropositive cases which the automated tests had failed to detect were detected by the manual tests. It was concluded that a combination of the ART and automated Reiter protein complement fixation test (ARPCFT) would be ideal for use in a large-scale screening programme for the detection of syphilis.

Previous authors (Pugh and Gaze, 1966; Wagstaff et al, 1969) have reported favourably on the use of the Technicon AutoAnalyzer for automation of the CWR and RPCFT, but one of the main problems seemed to be the continuous flow system's limited rate of testing. If all specimens were tested against cardiolipin and Reiter antigens separately, and all those giving positive or doubtful results were retested against complement alone, it would be difficult to test more than 150 samples each day as allowance must be made for the time required to prepare the machine for use, to wash it through after testing is finished, and to transcribe the results. This would not be considered a significant improvement on the manual test. It is, of course, possible to

Received for publication 14 October 1975 double the output of the AutoAnalyzer by using a two-channel system consisting of two samplers, a double set of reaction coils, and a two-pen recorder, as described by Wagstaff et al (1969), but this would increase the work involved in testing and add to the cost of an already expensive piece of equipment. A proposed alternative is to use a single-channel system and screen with a cardiolipin and Reiter antigen pool, testing fully only those specimens which register as positives. This, together with other minor improvements, should effectively double the output of the AAII system and, if used in conjunction with the ART, should be suitable for use in large-scale screening programmes.

\section{Material and Methods}

Of the samples received for testing, $80 \%$ were sent 
from venereal disease clinics, the remaining $20 \%$ consisting of antenatal specimens and sera from hospitals and general practitioners submitted for routine investigation or to comply with emigration requirements. Most were sent as clotted blood and these were separated by centrifugation at 1000 $\mathrm{rev} / \mathrm{min}$ for 10 minutes and the sera were decanted and stored at $4^{\circ} \mathrm{C}$ until required. Sera containing particulate matter were clarified by passage through a Hemming's filter. All sera were inactivated by heating at $56^{\circ} \mathrm{C}$ for 30 minutes on the day before testing and again on the morning of the test. Each serum specimen was then divided into two equal volumes, one for manual testing and the other for automated tests.

MANUAL TESTS

The VDRL slide test, CWR, RPCFT, and FTA-ABS tests were all performed according to the methods described in the Laboratory Diagnosis of Venereal Diseases (Wilkinson et al, 1972).

\section{AUTOMATED REAGIN TEST}

The ART was performed according to the method of McGrew et al (1968), using the Technicon AutoAnalyzer (AAII), with the exception that in this instance all samples were inactivated before testing. A pilot study on 20 known positive sera carried out before this investigation did not reveal any differences in titre between inactivated and uninactivated sera, and therefore to simplify procedure all sera were inactivated before testing. The tests were carried out at a room temperature of $25^{\circ} \mathrm{C}$ when possible, using Searle carbon antigen. Serum samples were tested in batches of 10 , separated by a vegetable dye marker to facilitate the location of specimens on the filter paper strip. Weak positive controls were tested at the beginning and end of each run to monitor sensitivity. Any positives detected by the machine were subsequently titrated, so that a quantitative estimate of antibody levels could be reported. To measure the effect of temperature on the reaction some specimens were run at $25^{\circ} \mathrm{C}$ and then with antigen heated to $37^{\circ} \mathrm{C}$. After all testing had been completed the glass reaction coils and connecting tubing were washed through with a $1 / 10$ dilution of Decon 90 followed by a 30-minute wash with deionized water.

AUTOMATED COMPLEMENT FIXATION TEST The cardiolipin and Reiter complement fixation tests were carried out on the Technicon AutoAnalyzer using the manifold depicted in fig 1 and based on the original method of Pugh and Gaze (1966).

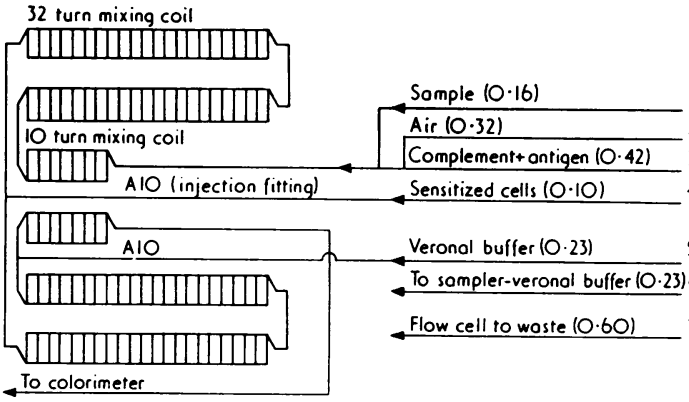

Fig 1 Automated complement fixation test. Figures in parentheses represent flow rates $(\mathrm{ml} / \mathrm{min})$

\section{COMPLEMENT HAEMOLYSIN CHESSBOARD} TITRATION

Using sheep blood preserved in Alsever's solution (Oxoid), a $2 \%$ of cell suspension was prepared as $>$ follows: the cells were washed three times in veronal buffer by repeated centrifugation at $1000 \mathrm{rev} / \mathrm{min} . \vec{\omega}$ If after three washes the supernatant was not clear (a rare occurrence) the cells were discarded. Provided the supernatant was clear, a $1.0 \mathrm{ml}$ volume of washed cells was mixed with $25 \mathrm{ml}$ of veronal buffer. One millilitre of this suspension was then removedo and placed in a haematocrit tube. The cells wereő

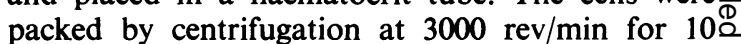
minutes and the volume of the veronal buffer in the $\overrightarrow{\overrightarrow{0}}$ $25 \mathrm{ml}$ suspension was adjusted according to the 3 haematocrit reading to give a $2 \%$ suspension. Ten dilutions $(1 / 500,1 / 1000 \ldots 1 / 5000)$ of rabbit haemo-․․ lytic serum (Wellcome) were prepared in veronalo buffer, and $1.0 \mathrm{ml}$ of each dilution was transferred to 10 separate tubes containing $1 \mathrm{ml}$ of a $2 \% \mathrm{RBC} 3$. suspension, thus giving ten $1 \%$ cell suspensions sensitized with dilutions of haemolysin ranging from $1 / 1000$ to $1 / 10000$. These tubes were theno incubated at $37^{\circ} \mathrm{C}$ for 10 minutes. Freeze-dried complement (Wellcome) was reconstituted accordingo to the manufacturer's instructions and 10 dilutions $(1 / 200,1 / 210 \ldots 1 / 290)$ were prepared in veronalos buffer. Using a vegetable dye as a marker, the timen taken for the complement to travel from the begin-N ning of line 3 through the first part of the reactioncoil to the RBC injection point (fig 1) was measured By the same method the time taken for the RBCs to travel from the start of line 4 to the injection pointe was also measured. The difference between the two gave the time lapse required between starting thes complement and starting the sensitized cells to $\vec{Q}$ ensure their simultaneous arrival at the injections point. Each complement dilution was then pumpeof through line 3 for 11 minutes starting with the lowesk dilution. After the appropriate time lapse (18 ming 
$40 \mathrm{~s}$ in this case) the RBCs sensitized with varying haemolysin dilutions were pumped through line 4 . Each dilution was pumped for 1 minute, giving a total time of 10 minutes to sample all the dilutions. A time lapse of 1 minute was allowed between each set of haemolysin dilutions to separate them and keep the titration in phase. A complete set of haemolysin dilutions was pumped through for every complement dilution. The pen recorder was switched on 35 minutes after the start of the titration, and the results were obtained by consulting the chart. Complete haemolysis is indicated by a continuous line on the chart at some point near the base, depending on the colorimeter setting. Unlysed cells will cause a deflection towards the top of the chart. The highest dilution of haemolysin and complement which, in reacting together, gave an unbroken line ( $100 \%$ lysis) on the chart were regarded as 1 minimum haemolytic dose (MHD). Both reagents were used at $1 \frac{1}{4}$ MHD in the test.

ANTIGEN ANTIBODY CHESSBOARD TITRATION Dilutions of both Reiter (BBL) and cardiolipin (Wellcome) antigen were prepared in $1 \frac{1}{4} \mathrm{MHD}$ of complement, the Reiter from $1 / 150$ to $1 / 250$ and the cardiolipin from $1 / 200$ to $1 / 800$. An appropriate range of doubling dilutions of a known positive serum was made in veronal buffer. The first dilution of Reiter antigen + complement was pumped through line 3 (fig 1) for 5 minutes while the complete set of positive serum dilutions was pumped through the sample line. This was repeated for all the Reiter antigen dilutions. The cardiolipin antigen was titrated in the same way. After the appropriate time lapse, sensitized cells were pumped through line 4. The pen recorder was switched on and the chart was consulted to determine the antigen end points. These were selected as the highest dilution which gave an optimum antibody titre.

\section{ANTIGEN COMPLEMENT TITRATION}

To determine whether or not the Reiter or cardiolipin antigens were anticomplementary, titrations were carried out on each batch as follows: complement dilutions in the region of 1 MHD were prepared, and antigen at the dilution in which it was to be used in the test was added to each complement dilution. The complement was then titrated against $1 \frac{1}{4}$ MHD of haemolysin by pumping the different complement + antigen dilutions through line 3 for 3 minutes each and the haemolysed cells through line 4 after the appropriate time interval. The results were recorded on the chart. If the antigens were anticomplementary the complement titre was adjusted according to the results of this test.
TEST PROCEDURE

Sera to be tested were arranged in a carefully checked, predetermined order in batches of 10 on the sampler heads. Complement was pumped through line 3 for 30 minutes to neutralize any residual anticomplementary activity due to the Decon 90 wash solution. Veronal buffer was pumped through all other lines. The colorimeter and pen recorder were switched on for a preliminary warm-up period. After the reaction coils had been washed through with complement, a mixture of cardiolipin and Reiter antigen at the correct titre diluted in $1 \frac{1}{4}$ MHD of complement was pumped through line 3 . The sampler was then switched on and serum samples were pumped through line 1. After the appropriate time lapse sensitized cells were pumped through line 4 , and 16 minutes later the pen recorder was switched on and readings were taken. All sera which gave a deflection of 20 transmission lines (TL) or greater were repeated against complement, cardiolipin antigen, and Reiter antigen separately to determine whether the deflection was due to anticomplementary activity or to the presence of specific antibodies. The reading against complement alone was subtracted from the reading against the complement antigen mixture, and if the difference was less than $20 \mathrm{TL}$, it was regarded as negative; a figure of 20-30 TL was regarded as weak positive, and greater than $30 \mathrm{TL}$ as positive. After testing had been completed all lines except the one to the wash receptacle were washed through with a $1 / 10$ dilution of Decon 90 for 15 minutes and rinsed with deionized water for 30 minutes.

\section{Results}

The results obtained in this comparative study are summarized in table $I$. Of the 7843 sera tested in parallel by the manual and automated methods, 258 gave positive results with one or more of the screening tests, 117 of them giving the same positive result by both methods. There were thus 141 sera which gave conflicting results. The ART detected 73 positives and six false positives, and failed to detect eight specimens ('false negatives') subsequently found to be positive. The corresponding figures for the VDRL slide test were 71 positives, 17 false positives, and 10 false negatives. The ARPCFT recorded 127 positives, 16 false positives, and only two false negatives, in contrast with the manual RPCFT, which detected only 83 positives and 11 false positives while recording as many as 46 false negatives. This position was the reverse of that found with the CWR, the scores for the automated test being 60 positives, 21 false positives, and 26 
false negatives, and for the manual test 82 positives, 36 false positives, and four false negatives.

The reproducibility of the automated complement fixation test results was measured by repeat testing of control sera. The results are shown in table II. The overall standard deviation was calculated as $\pm 2.5 \mathrm{TL}$.

A comparison between VDRL titres and ART titres using heated and cold antigen is shown in table III. An overall increase in sensitivity was achieved by maintaining the carbon antigen at $37^{\circ} \mathrm{C}$.

Several cases of syphilis were detected by only one

\begin{tabular}{lcccc}
\hline Test & $\begin{array}{l}\text { True } \\
\text { Positive }^{1}\end{array}$ & $\begin{array}{l}\text { Borderline } \\
\text { FTA-ABS }\end{array}$ & $\begin{array}{l}\text { False } \\
\text { Positive }\end{array}$ & $\begin{array}{l}\text { False } \\
\text { Negative }\end{array}$ \\
\hline ART & 73 & 1 & 6 & 8 \\
VDRL & 71 & 1 & 17 & 10 \\
ARPCFT & 127 & 12 & 16 & 2 \\
RPCFT & 83 & 7 & 11 & 46 \\
ACWR & 60 & 0 & 21 & 26 \\
CWR & 82 & 4 & 36 & 4 \\
\hline
\end{tabular}

Table I Comparison of results obtained by manual and automated methods in the screening of 7843 sera

${ }^{1}$ True positive sera were regarded as those which gave an FTA-ABS result of + or greater.

\begin{tabular}{llll}
\hline $\begin{array}{l}\text { Number } \\
\text { of } \\
\text { Test }\end{array}$ & Control Serum & & \\
\cline { 2 - 3 } & 1 & 2 & 3 \\
& $T L$ readings & $T L$ readings & $T L$ readings
\end{tabular}

$\overline{A C W R}$ ARPCFT $\overline{A C W R}$ ARPCFT $\overline{A C W R}$ ARPCFT

\begin{tabular}{lllllll}
\hline 1 & 15 & 65 & 38 & 84 & 70 & 42 \\
2 & 16 & 68 & 36 & 89 & 70 & 41 \\
3 & 15 & 63 & 36 & 81 & 69 & 42 \\
4 & 15 & 69 & 37 & 82 & 70 & 42 \\
5 & 14 & 65 & 34 & 85 & 75 & 41 \\
6 & 16 & 64 & 38 & 84 & 73 & 42 \\
7 & 16 & 63 & 36 & 84 & 70 & 42 \\
9 & 15 & 61 & 37 & 86 & 73 & 40 \\
$\begin{array}{l}\text { Standard } \\
\text { deviation }\end{array}$ & 15 & $\mathbf{6 4} \cdot 73$ & $36 \cdot 5$ & $84 \cdot 4$ & $71 \cdot 25$ & $41 \cdot 5$ \\
$\begin{array}{l}\text { Average } \\
\text { standard }\end{array}$ & $=3 \cdot 76$ & $\pm 1 \cdot 85$ & \pm 3.86 & $\pm 3 \cdot 0$ & \pm 0.43 \\
deviation & & & & & & \\
\hline
\end{tabular}

Table II Consistency of results obtained in automated CFT when repeat testing same specimens

\begin{tabular}{llll}
\hline $\begin{array}{l}\text { Patient } \\
\text { No. }\end{array}$ & $\begin{array}{l}\text { VDRL } \\
\text { Titre }\end{array}$ & $\begin{array}{l}\text { ART Titre } \\
\text { Room Temp. }\end{array}$ & $\begin{array}{l}\text { ART Titre } \\
\text { Antigen at } 37^{\circ} \mathrm{C}\end{array}$ \\
\hline 5082 & $1: 4$ & $1: 8$ & $1: 16$ \\
1410 & Neat & $1: 2$ & $1: 4$ \\
8605 & Neat & Neat & $1: 2$ \\
9309 & Neat & $1: 4$ & $1: 4$ \\
515 & $1: 32$ & $1: 32$ & $1: 64$ \\
2589 & Neat & $1: 2$ & $1: 4$ \\
2064 & Neat & Neat & Neat \\
8054 & $1: 2$ & $1: 8$ & $1: 16$ \\
\hline
\end{tabular}

Table III Effect of temperature on the sensitivity of the ART

of the screening antigens. These are depicted as single antigen positives (table IV) and give some measure of the relevant importance of each antigen. $\overrightarrow{\vec{F}}$ The manual test detected four single antigen posi- $-\frac{0}{-}$ tives, two Reiter and two VDRL which failed to $\frac{\bar{C}}{\sigma}$ register in any of the automated tests, compared $\overline{\bar{c}}$. with a total of 21 single antigen positives, 17 Reiter,, $\overrightarrow{\mathbb{D}}$ and four ART which failed to register in any of the manual tests.

\begin{tabular}{llll}
\hline Test & ART/VDRL & $\begin{array}{l}\text { Reiter } \\
\text { Antigen }\end{array}$ & $\begin{array}{l}\text { Cardiolipin } \\
\text { Antigen }\end{array}$ \\
\hline Automated & 4 & 17 & 0 \\
Manual & 2 & 2 & 0
\end{tabular}

Table IV Single antigen positives, ie, treponemal infections in which only one test was positive

\section{Discussion}

The ART compared favourably with the manual VDRL slide test, detecting a greater number of positives and fewer false positives; in fact, of theo 7843 sera tested, only six gave a false positive ART result, a surprising degree of specificity for a testo which uses a cardiolipin antigen. The ARPCFTO emerged as the most sensitive single screening test, $\stackrel{\mathbb{Q}}{2}$ detecting a total of 127 positives, of which 17 were single antigen positives. This was a considerables improvement on the 83 positives detected by the manual RPCFT. The increased sensitivity of the ARPCFT resulted in a corresponding increase in the number of false positives detected by this test:If the borderline FTA-ABS results are included as 3 . false positives, this gives a total of 28 out of the 7843 . sera tested, which is approximately double the rate for the RPCFT reported by Sequeira (1962). But, ino performing the ARPCFT, we deliberately sacrificed some degree of specificity for increased sensitivityo as the test, like the others in this comparative study was being used to screen for seropositive cases whichos were later to be checked by the FTA-ABS test. The results obtained with the cardiolipin complemen fixation test represent an anomaly which is difficulto to explain. The automated complement fixation test had been carefully designed to give maximum sensitivity, and the results obtained with the Reiteres antigen seem to indicate that this objective had been achieved. The failure to achieve a corresponding increase in sensitivity with the CWR was extensively investigated but no adequate explanation was found $\overrightarrow{\mathrm{D}}$ It is, however, interesting to note that the increase of sensitivity of the manual CWR did not result in the detection of a greater number of positive cases ob 
syphilis; in fact if the CWR had been excluded from this comparative study, not one case of treponemal infection would have been missed by the manual or automated tests and 57 false positive results would have been avoided. These results tend to support the opinion expressed by previous authors (Wilkinson et al, 1972; Schofield, 1973) that the CWR is superfluous in a screening system which incorporates a cardiolipin flocculation test and only adds to the number of false positive results obtained. Omitting the CWR from the automated screening test would simplify the complement fixation test by reducing the number of repeat tests necessary on positives detected by a pooled antigen.

The AutoAnalyzers functioned efficiently throughout this investigation and have continued to do so since. The feared 'carry-over phenomenon' (ie, the 'contamination' of a negative specimen by trailing traces of a strongly positive serum passing ahead of it through the circuit), which is one of the

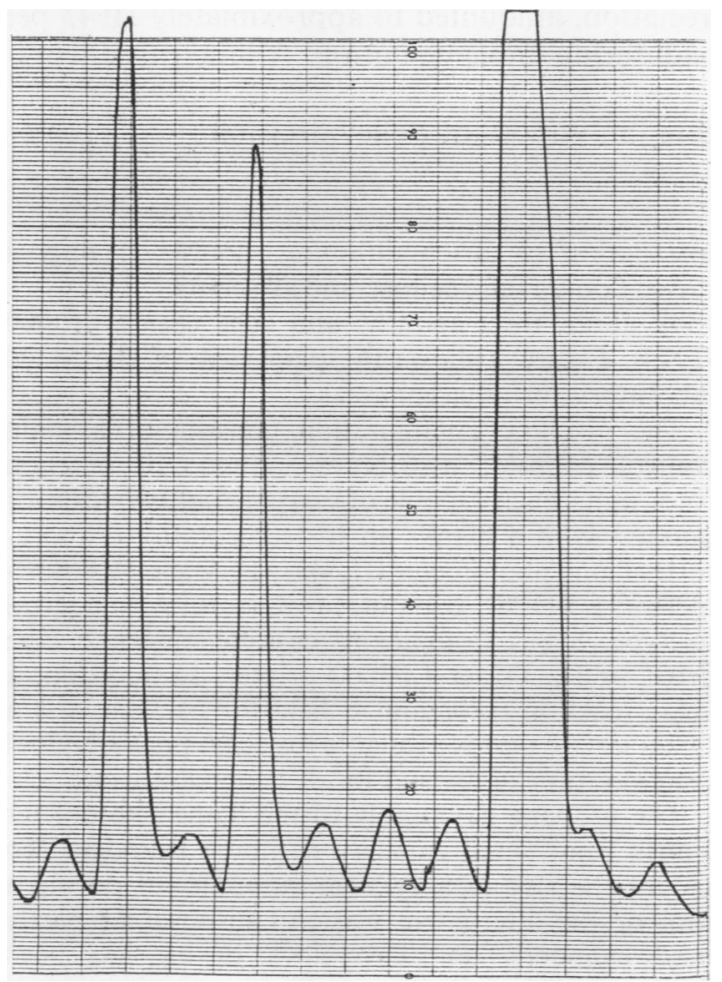

Fig 2 Sample of chart from AAII recorder showing good wash characteristics of system, ie, no carryover from positive samples to following negative samples recognized theoretical drawbacks of the continuousflow system of automation, was rarely encountered as the wash characteristics of the equipment are very good (fig 2). As an added safeguard, whenever two successive specimens were found to be positive the second was always retested with known negative sera in the cups on either side of it in the sampler.

\section{Conclusion}

In the comparison with the standard manual tests the ART and ARPCFT showed greater sensitivity which resulted in the detection of an increased number of treponemal infections. The ACWR did not compare favourably with its manual counterpart but fortunately this was of no consequence as it was concluded that the CWR was irrelevant in the context of a screening system which included the ART. It therefore appears that the ideal system for largescale screening for syphilis should consist of the ART and ARPCFT with the FTA-ABS as the final arbiter. Such a system would have the capacity for testing and reporting 400 samples per day and could be operated by one skilled member of staff. This capacity should meet the requirements of most large-scale screening centres but it could be increased by using a dual system if necessary. A largescale automated screening system would have the additional advantages of daily reporting, a considerable reduction in the cost per specimen, and, if required, quantitative results of proven reproducibility.

\section{References}

McGrew, B. E., Du Cros, M. J. F., Stout, G. W., and Falcone, V. H. (1968). Automation of a flocculation test for syphilis. Amer. J. clin. Path., 50, 52-59.

Pugh, V. W. and Gaze, R. W. T. (1966). The Reiter protein complement-fixation test using the AutoAnalyzer. J. clin. Path., 19, 595-599.

Schofield, C. B. S. (1973). Serological tests for syphilis in pregnancy. False and missed positive reactions. Brit. $J$. vener. Dis., 49, 420-426.

Sequeira, P. J. L. (1962). Serology of the venereal diseases. Brit. J. vener. Dis., 38, 9-18.

Wagstaff, W., Firth, R., Booth, J. R., and Bowley, C. C. (1969). Large-scale screening by the automated Wassermann reaction. J. clin. Path., 22, 236-239.

Wilkinson, A. E., Scrimgeour, G., and Rodin, P. (1972). A comparison of the absorbed fluorescent treponemal antibody (FTA-ABS) test and other screening tests for treponemal disease in patients attending a venereal disease clinic. J. clin. Path., 25, 437-440.

Wilkinson, A. E., Taylor, C. E. D., McSwiggan, D. A., Turner, G. C., Rycroft, J. A., and Lowe, G. H. (1972). Laboratory Diagnosis of Venereal Diseases. PHLS Monograph. HMSO, London. 